PROCEEDINGS OF THE

AMERICAN MATHEMATICAL SOCIETY

Volume 133, Number 11, Pages 3389-3394

S 0002-9939(05)07904-9

Article electronically published on June 7, 2005

\title{
AUTOMATIC DIFFERENTIABILITY AND CHARACTERIZATION OF COCYCLES OF HOLOMORPHIC FLOWS
}

\author{
FARHAD JAFARI, THOMAS TONEV, AND ELENA TONEVA
}

(Communicated by Juha M. Heinonen)

\begin{abstract}
In this paper we prove that cocycles of holomorphic flows on domains in the complex plane are automatically differentiable with respect to the flow parameter, and their derivatives are holomorphic functions. We use this result to show that, on simply connected domains, an additive cocycle is a coboundary if and only if this cocycle vanishes at the fixed point of the flow.
\end{abstract}

\section{INTRODUCTION}

On many occasions continuity and differentiability arise automatically if a function possesses some apparently unrelated properties. Exploitation of these properties has led to many deep results in functional analysis (see [Jar] and references therein, for example). This paper demonstrates another instance of such a result, leading to a complete and simple characterization of the multipliers of holomorphic semigroups of composition operators.

Let $\Omega$ be a domain (open, connected and nonempty) in the complex plane $\mathbb{C}$ and let $H(\Omega)$ be the set of holomorphic functions on $\Omega$. We denote by $\mathbb{D}$ the open unit disc in $\mathbb{C}$. A one-parameter family $\varphi(t, z):[0, \infty) \times \Omega \rightarrow \Omega$ of nonconstant holomorphic functions satisfying $\varphi(0, z)=z$ and $\varphi(s+t, z)=\varphi(s, \varphi(t, z))$ for all $s, t \geq 0$ and $z \in \Omega$ is called a holomorphic flow. We will consider continuous flows $\varphi(t, z)$ on $[0, \infty) \times \Omega$ only. We say $z_{0} \in \Omega$ is a fixed point for $\varphi$ if $\varphi\left(t, z_{0}\right)=z_{0}$ for all $t \geq 0$.

Note that $\varphi(t, z)$ are continuously differentiable with respect to $t$. Berkson and Porta $[\mathrm{BP}]$ have given an explicit formula for the derivative $\frac{\partial \varphi(t, z)}{\partial t}$ of a flow. Actually their argument proves the continuous differentiability of any holomorphic flow $\varphi(t, z)$ which is continuous with respect to $t$. Cowen [C] and Siskakis [S] give interesting applications of holomorphic flows to the study of Cesaro operators.

A continuous complex-valued function $m$ on $[0, \infty) \times \Omega$ is said to be a multiplicative cocycle for the flow $\varphi$ if $m$ satisfies the following conditions:

(i) $m(t, \cdot) \in H(\Omega)$ for all $t \geq 0$,

(ii) $m(0, z)=1$ for all $z \in \Omega$,

(iii) $m(t+s, z)=m(s, z) m(t, \varphi(s, z))$ for all $t, s \geq 0, z \in \Omega$.

Received by the editors March 7, 2003 and, in revised form, July 1, 2004.

2000 Mathematics Subject Classification. Primary 47D03; Secondary 47B38.

Key words and phrases. Flow, cocycle, infinitesimal generator. 
The family of weighted holomorphic composition operators $m(t, z) C_{\varphi(t, z)}$ is a strongly continuous semigroup on the Hardy spaces $H^{p}(\Omega)$ [EJ]. König ([Ko, Lemma 2.1 (b)) shows that every cocycle $m$ is nonvanishing and gives a characterization of the smooth cocycles on Hardy spaces of the unit disc.

Likewise, a continuous complex-valued function $a(t, z)$ on $[0, \infty) \times \Omega$ is said to be an additive cocycle for a holomorphic flow $\varphi$ if

(i') $a(t, z) \in H(\Omega)$ for all $t \geq 0$,

(ii') $a(0, z)=0$ for all $z \in \Omega$,

(iii') $a(t+s, z)=a(t, z)+a(s, \varphi(t, z))$ for $s, t \geq 0, z \in \Omega$.

If $a$ is an additive cocycle, then $m(t, z)=\exp (a(t, z))$ is a multiplicative cocycle and the converse is true for simply connected domains.

A (multiplicative) cocycle $m(t, z)$ is said to be a coboundary for the flow $\varphi$ if there exists a nonvanishing function $\alpha \in H(\Omega)$ such that

$$
m(t, z)=\frac{\alpha(\varphi(t, z))}{\alpha(z)} \text { for all }(t, z) \in[0, \infty) \times \Omega
$$

and, likewise, an (additive) cocycle $a(t, z)$ is said to be a coboundary for the holomorphic flow $\varphi$ if there exists a function $\beta \in H(\Omega)$ such that

$$
a(t, z)=\beta(\varphi(t, z))-\beta(z) \text { for all }(t, z) \in[0, \infty) \times \Omega .
$$

The utility and characterization of smooth cocycles which are coboundaries are presented in [JTTY]. The reader is referred to that paper for a detailed discussion of holomorphic flows and cocycles.

In this paper, we demonstrate that cocycles of holomorphic flows are automatically smooth and, using a characterization of such cocycles, provide a simple and complete characterization of cocycles which are coboundaries.

\section{MAIN RESULTS}

Lemma 1. Let a be an (additive) cocycle for a holomorphic flow $\varphi(t, z)$ on $\Omega$. Then

$$
\lim _{n \rightarrow \infty} 2^{n} a\left(\frac{1}{2^{n}}, z\right)=g(z) \in H(\Omega)
$$

where the convergence is uniform on compact subsets of $\Omega$.

Proof. Let $K$ be a compact subset of $\Omega$, and let $r>0$ be such that $2 r<\operatorname{dist}(K, b \Omega)$ $=\inf \{|z-w|: z \in K, w \in b \Omega\}$. Denote by $K_{2 r}$ the compact set $K_{2 r}=\{z \in \mathbb{C}:$ $\operatorname{dist}(z, K) \leq 2 r\} \subset \Omega$.

Since $z=\varphi(0, z)$ and $\varphi$ is uniformly continuous on $[0,1] \times K$, we can find a constant $c>0$, such that $|z-\varphi(t, z)| \leq r$ for all $t \leq c$ and for every $z \in K$. We will use some ideas from the proof of Theorem 1.1 in [BP]. Let $[z, \varphi(t, z)]$ be the segment in $\mathbb{C}$ connecting $z$ and $\varphi(t, z)$, and let $C_{\zeta}$ be the circle with radius $2 r$ centered at $\zeta$. Clearly, dist $\left(C_{\zeta},[z, \varphi(t, z)]\right) \geq r$ for every $t \in[0, c], \zeta \in[z, \varphi(t, z)]$, and

$$
\begin{gathered}
a(2 t, z)-2 a(t, z)=a(t, \varphi(t, z))-a(t, z) \\
=\int_{z}^{\varphi(t, z)} \frac{\partial a(t, \zeta)}{\partial \zeta} d \zeta=\int_{z}^{\varphi(t, z)} \frac{1}{2 \pi i}\left(\int_{C_{\zeta}} \frac{a(t, \xi)}{(\xi-\zeta)^{2}} d \xi\right) d \zeta,
\end{gathered}
$$


by the Cauchy theorem for derivatives of holomorphic functions. Therefore,

$$
|a(2 t, z)-2 a(t, z)| \leq \max _{[0, c] \times K_{2 r}}|a(t, z)| \frac{r}{r^{2}}|z-\varphi(t, z)| .
$$

Observe that $K_{2 r} \supset C_{\zeta}$ for every $\zeta \in[z, \varphi(t, z)]$ and every $z \in K$. Since $\varphi$ is continuously differentiable in the $t$ variable, we can find a constant $C>0$, such that

$$
|z-\varphi(t, z)|=|\varphi(0, z)-\varphi(t, z)| \leq C t
$$

for every $z \in K$ and $t \in[0, c]$. Consequently, for any $t \in[0, c]$ and any $z \in K$,

$$
|a(2 t, z)-2 a(t, z)| \leq \max _{[0, c] \times K_{2 r}}|a(t, z)| \frac{C}{r} t=N t
$$

where $N=\max _{[0, c] \times K_{2 r}}|a(t, z)| \frac{C}{r}>0$.

Now

$$
\begin{aligned}
|a(t, z)|=\frac{1}{2} \mid(2 a(t, z) & -a(2 t, z))+a(2 t, z) \mid \leq \frac{1}{2}(N t+|a(2 t, z)|) \\
& \leq \frac{1}{2} N t+\frac{|a(2 t, z)|}{(2)^{2 / 3}} .
\end{aligned}
$$

Hence, for the function $\psi(t, z)=\frac{a(t, z)}{t^{2 / 3}}$ we have

$$
|\psi(t, z)| \leq \frac{1}{2} N t^{1 / 3}+\frac{|a(2 t, z)|}{(2 t)^{2 / 3}}=\frac{1}{2} N t^{1 / 3}+|\psi(2 t, z)|
$$

for any $t \in(0, c]$ and any $z \in K$.

We claim that $\psi(t, z)$ is bounded on $(0,1] \times K$. Indeed, without loss of generality we can assume that $c<1$. By continuity, there are constants $L$ and $\eta \leq c$, such that $|\psi(t, z)| \leq L$ for $t \in[\eta, \max \{1,2 \eta\}]$ and $z \in K$. Now (4) implies that $|\psi(t, z)| \leq(1 / 2) N c^{1 / 3}+L$ for $z \in K$ and for $t \in[\eta / 2, \eta]$ (and, consequently, for $t \in[\eta / 2,1]$ too). By using (4) again, we extend the $t$-interval closer to 0 for the expense of an additional term in the upper bound. Namely,

$$
|\psi(t, z)| \leq \frac{1}{2} N c^{1 / 3}+\frac{1}{2} N\left(\frac{c}{2}\right)^{1 / 3}+L
$$

for any $t \in[\eta / 4,1]$ and $z \in K$. Proceeding inductively, we obtain that

$$
\left|\frac{a(t, z)}{t^{2 / 3}}\right|=|\psi(t, z)| \leq \frac{1}{2} N c^{1 / 3} \sum_{n=1}^{\infty}\left(\frac{1}{2^{n}}\right)^{1 / 3}+L=\frac{1}{2} N c^{1 / 3} \sum_{n=1}^{\infty} \frac{1}{\left(2^{1 / 3}\right)^{n}}+L=M
$$

for any $t \in(0,1]$ and $z \in K$. Note that the geometric series from the above is convergent and its sum is independent of both $t$ and $z$.

Consequently, for every compact set $K \subset \Omega$ there is a constant $M$, such that

$$
|a(t, z)| \leq M t^{2 / 3}
$$

for every $t \in[0,1]$ and $z \in K$. In particular, there is a constant $M_{2 r}$ such that (5) holds on the set $K_{2 r}$.

By applying (5) we refine the estimate from (1). We find a positive constant $c$, such that for every $t \in[0, c]$ and $z \in K$,

$$
|2 a(t, z)-a(2 t, z)| \leq M_{2 r} t^{2 / 3} C t=\widetilde{M} t^{5 / 3},
$$


for some constant $\widetilde{M}$. Assume that $t \in(0, c]$. Therefore, by dividing both sides of (6) by $2 t$, we get

$$
\left|\frac{a(t, z)}{t}-\frac{a(2 t, z)}{2 t}\right| \leq \frac{\widetilde{M} t^{2 / 3}}{2} .
$$

For $n$ big enough, so that $t=1 / 2^{n} \leq c$, we have

$$
\left|2^{n} a\left(\frac{1}{2^{n}}, z\right)-2^{n-1} a\left(\frac{1}{2^{n-1}}, z\right)\right| \leq \frac{1}{2} \widetilde{M} \frac{1}{\left((2)^{2 / 3}\right)^{n}} .
$$

The Weierstrass domination theorem implies that the series

$$
a(1, z)+\sum_{n=1}^{\infty}\left[2^{n} a\left(\frac{1}{2^{n}}, z\right)-2^{n-1} a\left(\frac{1}{2^{n-1}}, z\right)\right]
$$

is uniformly convergent on $K$ to a function $g(z) \in C(K)$. Consequently, the sequence $2^{n} a\left(\frac{1}{2^{n}}, z\right)$ is uniformly convergent to $g(z)$ on compact subsets of $\Omega$, as claimed. Clearly, $g$ is holomorphic in $\Omega$.

Theorem 2. Every additive cocycle $a(t, z)$ for a holomorphic flow $\varphi$ admits the representation

$$
a(t, z)=\int_{0}^{t} g(\varphi(s, z)) d s,
$$

where $g$ is the function in Lemma 1.

Proof. Indeed,

$$
\begin{aligned}
\int_{0}^{t} g & \varphi(s, z)) d s=\int_{0}^{t} \lim _{n \rightarrow \infty} 2^{n} a\left(\frac{1}{2^{n}}, \varphi(s, z)\right) d s \\
& =\lim _{n \rightarrow \infty} 2^{n} \int_{0}^{t} a\left(\frac{1}{2^{n}}, \varphi(s, z)\right) d s=\lim _{n \rightarrow \infty} 2^{n} \int_{0}^{t}\left[a\left(\frac{1}{2^{n}}+s, z\right)-a(s, z)\right] d s \\
& =\lim _{n \rightarrow \infty} 2^{n}\left[\int_{1 / 2^{n}}^{t+1 / 2^{n}} a(s, z) d s-\int_{0}^{t} a(s, z) d s\right] \\
& =\lim _{n \rightarrow \infty} \frac{1}{1 / 2^{n}}\left[\int_{t}^{t+1 / 2^{n}} a(s, z) d s-\int_{0}^{1 / 2^{n}} a(s, z) d s\right]=a(t, z)-a(0, z)=a(t, z)
\end{aligned}
$$

by the Mean Value Theorem for integrals and the continuity argument.

Theorem 3. If $\Omega$ is a simply connected domain in the complex plane $\mathbb{C}$ and $m$ is a multiplicative cocycle on $[0, \infty) \times \Omega$, then there is a holomorphic function $g: \Omega \rightarrow \mathbb{C}$, so that

$$
m(t, z)=\exp \left(\int_{0}^{t} g(\varphi(s, z)) d s\right) .
$$

Proof. Simply recall that $m(t, z)=\exp (a(t, z))$. 
Differentiating $m$ expressed in the above theorem provides an explicit expression for the generator $g(\varphi(t, z))=\frac{\partial a(t, z)}{\partial t}$ of the additive cocycle $a(t, z)$ related to $m$. Since $m$ is nonvanishing, we have

$$
g(\varphi(t, z))=\frac{1}{m(t, z)} \frac{\partial m(t, z)}{\partial t} .
$$

By Lemma 2.2 in [JTTY], the flow is a univalent holomorphic function for every $t \geq 0$. Therefore, for such domains, $g$ takes the explicit form

$$
g(z)=\frac{1}{m(t, w)} \frac{\partial m(t, w)}{\partial t} \text { where } w=\varphi^{-1}(t, z) .
$$

By applying locally the argument from the proof of Theorem 3 we immediately obtain the following.

Theorem 4. Each multiplicative cocycle $m(t, z)$ on a connected domain $\Omega \subset \mathbb{C}$ is continuously differentiable with respect to the flow parameter $t$, and its derivative is holomorphic on $\Omega$.

Theorem 5. Let $\Omega$ be a simply connected domain in $\mathbb{C}$ and let $\varphi:[0, \infty) \times \Omega \rightarrow \Omega$ be a holomorphic flow. Let a be an (additive) cocycle and $m$ be the corresponding (multiplicative) cocycle for $\varphi$.

(i) If the flow is fixed-point-free, then every cocycle is a coboundary.

(ii) If the flow has a fixed-point at $z_{0} \in \Omega$, then a (resp. $m$ ) is a coboundary if and only if $a\left(t, z_{0}\right)=0$ (resp. $\left.m\left(t, z_{0}\right)=1\right)$ for all $t \geq 0$.

Proof. Let $G$ denote the infinitesimal generator of $\varphi(t, z)$, i.e., $G$ is the solution of the initial value problem

$$
\frac{\partial \varphi(t, z)}{\partial t}=G(\varphi(t, z)), \varphi(0, z)=z \text { for all } z \in \Omega
$$

(i) If $a$ is an additive cocycle for the fixed-point-free flow $\varphi, G(z) \neq 0$, and since $a$ is continuously differentiable in $t$ by Theorem 2, the result follows from Theorem 4.2 in [JTTY, p. 248].

(ii) Suppose the flow $\varphi$ has a fixed-point at $z_{0} \in \Omega$. Then from the integral representation for $a$ in Theorem 2 ,

$$
\frac{\partial a(t, z)}{\partial t}=g(\varphi(t, z))
$$

which implies that $a\left(t, z_{0}\right)=\operatorname{tg}\left(z_{0}\right)$, since $a(0, z)=0$. Now, by Theorem 4.3 (ii) in [JTTY, p. 249], since $G\left(z_{0}\right)=0$, it follows that $a$ is a coboundary if and only if $g\left(z_{0}\right)=0$. By continuity, this clearly implies $a\left(t, z_{0}\right)=0$ for every $t \geq 0$.

\section{OPEN PROBLEMS}

It would be interesting to investigate higher order of differentiability with respect to $t$. The most natural generalization of this paper would involve studying holomorphic flows on multiply connected domains in the complex plane and identifying the structure of the cocycles acting on semigroup flows on such domains. For example, it would be very interesting to provide a classification of semigroup flows on annular domains and characterize the cocycles which are coboundaries on such domains. 
Replacing the nonvanishing holomorphic functions as the coefficient group by more restricted coefficient groups such as the class of boundedly invertible holomorphic functions on $G$ or, more generally, by the algebra of bounded holomorphic functions on $G$ would clarify the structure of semigroup flows on these spaces.

As an immediate extension of this paper, it would be interesting to know if projective cocycles (see [JTTY, Example 5.1 and Proposition 5.2]) are also automatically differentiable. This will help answer the question: Given $h \in H(G)$ can one find a nonvanishing weight function $p$ such that $m(t, z)=p(t) \exp (\operatorname{th}(z))$ is a cocycle?

Applications of the structure of cocycles of holomorphic flows to dynamical systems and semigroup theory would also be of interest.

\section{ACKNOWLEDGMENT}

The authors gratefully acknowledge helpful comments and valuable improvements by the referee.

\section{REFERENCES}

[BP] E. Berkson and H. Porta, Semigroups of analytic functions and composition operators, Michigan Math. J. 25(1978), 101-115. MR0480965 (58:1112)

[C] C. C. Cowen, Subnormality of the Cesaro operator and a semigroup of composition operators, Indiana Univ. Math. J. 33 (1984), 305-318. MR0733903 (86g:47034)

[EJ] J.-Cl. Evard and F. Jafari, On semigroups of operators on Hardy spaces, preprint (1995).

[JTTY] F. Jafari, T. Tonev, E. Toneva, K. Yale, Holomorphic flows, cocycles and coboundaries, Michigan Math. J. 44 (1997), 239-253. MR1460412 (99c:47050)

[Jar] K. Jarosz, Automatic continuity of separating linear isomorphisms, Canad. Math. Bull. 33 (1990), 139-144. MR1060366 (92j:46049)

[Ko] W. König, Semicocycles and weighted composition semigroups on $H^{p}$, Michigan Math. J. 37 (1990), 469-476. MR.1077330 (91m:47057)

[S] A. G. Siskakis, Composition semigroups and the Cesaro operator on $H^{p}$, J. London Math. Soc. (2) 36 (1987), 153-164. MR0897683 (89a:47048)

Department of Mathematics, University of Wyoming, Laramie, Wyoming 82071-3036

E-mail address: fjafari@uwyo.edu

Department of Mathematical Sciences, University of Montana, Missoula, Montana $59812-1032$

E-mail address: tonevtv@mso.umt.edu

Department of Mathematics, 216 Kingston Hall, Eastern Washington University, Cheney, Washington 99004-2418

E-mail address: etoneva@mail.ewu.edu 